faschistischen „Alleanza Nazionale“ und die Positionen der pro-faschistischen „Casa Pound“ in Italien. So nehmen die Autoren politische Prozesse in vielen Ländern aus der journalistischen Perspektive in den Blick, womit gemeint ist, dass persönliche Eindrücke, Beobachtungen und Hintergrundgespräche mit Experten zu den jeweiligen Themen den Kernbestand der einzelnen Länderkapitel ausmachen.

Bilanzierend heißt es: „Vierzehn europäische Länder, ein internationaler Ort. Die radikale Rechte bemüht sich, gesellschaftliche Akzeptanz zu gewinnen. Auf den Straßen protestieren Parteien und Kameradschaften gegen Sozialkürzungen und Rentenaltererhöhung. In den Parlamenten, auf Landes-, nationaler oder europäischer Ebene, lehnen die Abgeordneten und Landtagsmandatsträger Einwanderung und Bankenunterstützung $a b$, und bei Konzerten beklagen Bands und Fans den Bau von Moscheen und Identitätsverluste. Themen nicht nur am rechten Rand der Gesellschaften in Europa. In den vergangenen Jahren sind in den Staaten, so belegen es Studien, rechte Ressentiments gewachsen. Die Themen der radikalen Rechten sind die Themen der nervösen Gesellschaften ...“ (S. 269) „So unterschiedlich die Parteien und Kameradschaften in den Ländern erscheinen - ihnen allen gemein ist, die Ängste und Sorgen der Bürger auszunutzen, um Zuspruch und Wählergunst zu gewinnen" (S. 270). Einschlägige Einstellungen und ökonomische Krisenerfahrungen erklärten dies.

Das Urteil über das Buch ist abhängig vom Erwartungshorizont des Lesers: Wer eine gut lesbare eindringliche Darstellung zu Entwicklungen am „rechten Rand“ in den europäischen Ländern lesen möchte, ist hier mehr als nur gut bedient. Wer eine detaillierte Analyse mit genaueren Einschätzungen erwartet, wird wohl enttäuscht sein. Es handelt sich eben um ein journalistisches Buch. Da fangen Kapitel mit Sätzen wie „Der Saal ist kühl. Die Abendsonne wirft, nach einem sehr warmen Tag, noch einige Strahlen durch die Tür“ (S. 83) an. Es fehlt an genauen Belegen wie an näheren Informationen zu einzelnen Organisationen. Gleichwohl informiert der Band auch zur Entwicklung in Ländern im Umbruch wie Griechenland oder Ungarn, woraus aus der vergleichenden Perspektive etwas gelernt werden kann. Die Darstellung bleibt aber fast nur auf dieser phänomenologischen Ebene stehen. Die Frage nach der gesellschaftlichen Wirkung wird allzu einfach mit dem Hinweis auf andere Studien beantwortet.

Armin Pfahl-Traughber

\title{
Rechte Leute von links: historisch-biografische Einblicke und romanhafte Beschreibungen
}

Seitenbecher, Manuel: Mahler, Maschke \& Co. Rechtes Denken in der 68er-Bewegung?, Verlag Ferdinand Schöningh, Paderborn 2013, 557 Seiten, € 39,90.

Carini, Marco: Die Achse der Abtrünnigen. Über den Bruch mit der Linken, Rotbuch Verlag, Berlin 2012, 286 Seiten, € 14,95.

Das „Zeitalter der Extreme“ (Eric Hobsbawm) spiegelt sich nicht allein in der jüngeren Geschichte des gesamten europäischen Kontinents wider, sondern bisweilen auch in den Biografien Einzelner. Einen Teil von ihnen nimmt der Band „Mahler, Maschke \& Co.“ in den 
Blick. Darin wirft Manuel Seitenbecher die Frage auf, warum einzelne „68er“ (im Einzelnen: Tilman Fichter, Horst Mahler, Günter Maschke, Reinhold Oberlercher, Bernd Rabehl) im Laufe ihres Lebens einen radikalen politischen Lagerwechsel vollzogen haben (S. 12 f.). Horst Mahlers Stationen etwa lauten: SDS, RAF, NPD. Weil er - ebenso wie die anderen vier nicht müde wird, der eigenen Person ideologische Konstanz zu attestieren, erscheint die Frage Seitenbechers gar nicht so abwegig, ob überhaupt von einem ideologischen Wandel bei den fünf Protagonisten gesprochen werden kann. Um ihr nachzuspüren, verfolgt die in Potsdam erschienene Dissertation einen doppelten Vergleich: Der Autor konfrontiert einerseits die Protagonisten, andererseits die verschiedenen Lebensphasen miteinander.

Zum Vergleich der Lebensphasen: Seitenbecher fragt nach Wandel und Kontinuität im Leben der fünf Renegaten. Hierfür geht er die wichtigsten Etappen in chronologischer Reihenfolge durch: vom Sozialistischen Deutschen Studentenbund vor 1968 über die bewegte Zeit während der Studentenunruhen und das Auseinanderbrechen der „68er“ bis hin zur Gegenwart. Zu keinem Zeitpunkt unterliegt er der Versuchung, die fünf Auserwählten über den Status einer Ausnahme in dieser Kohorte hinauszuheben. Außerdem entlarvt seine faktengesättigte Studie die retrospektive, nationalrevolutionäre Deutung von 1968 durch die Protagonisten selbst als Umdeutung. Es handelt sich um Manöver, die öffentlichkeitswirksam der eigenen Person ideologische Konsistenz bescheinigen sollen. Allenfalls Bernd Rabehl könne mit einiger Berechtigung sagen, die Bahnen von damals nicht verlassen zu haben, denn er strebte schon in den 1960ern gemeinsam mit Rudi Dutschke nach der Eigenständigkeit Deutschlands - im Nachhinein freilich deutlich prononcierter als im Eifer des Geschehens selbst. Zudem liegen Welten zwischen seinem damaligen nationalrevolutionären Impetus und seinen heutigen politischen Ansichten.

Konstanten lassen sich bei den rechten Leuten von links dennoch identifizieren: Zeit ihres Lebens fielen sie etwa durch ihren radikaldemokratischen Impetus (Fichter / Rabehl) oder ihre marxistische Argumentationsweise (Oberlercher / Mahler) auf; desgleichen durch ihr ausgeprägtes Verlangen zu provozieren, durch ihre antiwestliche, parteien- und konsumkritische Haltung, schließlich durch ihr identitäres Verständnis von Volksherrschaft. Daher lesen sich ihre Biografien nur zum Teil als Geschichte einer politischen Konversion - nämlich die von links nach rechts. Ansonsten sind sie sich treu geblieben: Les extrêmes se touchent.

Zum Vergleich der fünf politischen Konvertiten: Die Ausführungen kratzen an der These Gerd Koenens und Wolfgang Kraushaars, es gebe so etwas wie eine „geschlossene Strömung" (S. 385), die vom linken zum rechten Rand gewechselt sei. Abgesehen von Reinhold Oberlercher und Horst Mahler, die ihre „völkisch-rassistische Reichsgläubigkeit“ (S. 386), ihr ausgeprägter Amerika- und Judenhass sowie ihre überdauernde Hegel-Anhängerschaft verbindet, sei das politische Konvertitentum nach 1968 von Heterogenität gekennzeichnet: So vereinnahmte etwa Günter Maschke die Chiffre „1968“ nie in derselben Intensität, wie es Bernd Rabehl, Reinhold Oberlercher und Horst Mahler tun, wenn sie im Rückblick nationalrevolutionäre Intentionen zu sehen glauben. Die beiden zuletzt genannten fallen ohnehin durch ihren heutigen Rassismus, ihren Bedrohungsnationalismus, insgesamt durch ihre kompromisslose Radikalität aus der Reihe; Mahler mehr noch als Oberlercher.

Gänzlich anders gelagert sei der Fall von Tilman Fichter. Er hebt sich von den anderen ab, weil er zu keinem Zeitpunkt radikal oder extremistisch gewesen sei. Das lässt indes die Frage aufkommen, warum er - SPD-Mitglied seit 1982 - überhaupt in die Untersuchung aufgenommen wird. Das Argument, er sah sich als einer der ersten dem Vorwurf 
ausgesetzt, seine „linke“ Herkunft verraten zu haben, weil er schon früh die deutsche Wiedervereinigung forderte, verfängt nicht. Es verweist nur auf die diffuse Vorstellung, die Seitenbecher (und mit ihm ein großer Teil der deutschen Politik und Publizistik) vom negativ konnotierten politischen Richtungsbegriff „,rechts“ hat (S. 32 - 34). Obendrein sitzt der Autor dem Missverständnis auf, Extremismus negiere den „gesellschaftlichen und politischen common sense" (S. 38). Er scheint sich zu scheuen, den Begriff zu gebrauchen, wo er geboten wäre. Dabei hätte er dazu beigetragen, das Untersuchungsdesign zu vereinheitlichen: Hätte Seitenbecher den Extremismusbegriff als Auswahlkriterium zugrunde gelegt, hätte sich seine Fallauswahl durch größere, die Analyse erleichternde Homogenität ausgezeichnet.

Einer noch größeren Heterogenität bei der Fallauswahl sieht sich ausgesetzt, wer „Die Achse der Abtrünnigen“ aufschlägt. Darin fragt der taz-Redakteur Marco Carini: „Aus welchen Motiven wenden sich Linke vom Kommunismus und Sozialismus ab? Und welche biografischen Konsequenzen, welche politischen Neuverortungen sind das Resultat dieser Abkehr?" (S. 8) Das Buch, weniger für den wissenschaftlichen „Elfenbeinturm“ als für ein breites, zeitgeschichtlich interessiertes Publikum geschrieben, widmet sich dem politischen Renegatentum von links. Obwohl hier kein wissenschaftlicher Anspruch besteht - es handelt sich, anders als bei Seitenbecher, nicht um eine Qualifikationsschrift -, spart Carini an den entscheidenden Punkten nicht an begrifflicher Klarheit: Dem Begriff „links“ ordnet er den Anspruch auf Verteilungsgerechtigkeit und auf unbedingte Chancengerechtigkeit zu (S. 14); mit dem Begriff „Renegat“ meint er jene, „die das politische Lager gewechselt haben, oft ohne dabei bestimmte Grundüberzeugungen aufzugeben“ (S. 12). Diese Definitionen sind zwar streitbar, taugen aber zur Verständigung.

Umso mehr überrascht allerdings die Auswahl, die Carini dem Renegatentum von links zurechnet. Sie schließt solche ein, die „nur“ mit dem Stalinismus gebrochen haben, dem Kommunismus beziehungsweise Sozialismus aber verhaftet geblieben sind (etwa Robert Havemann, Susanne Leonhard und Gerhard Zwerenz), zweitens Personen, die sich nach ihrem Wandel dem gemäßigt linken Spektrum verbunden fühlten (zum Beispiel Erich Loest), und drittens jene, die tatsächlich einen politischen Lagerwechsel vollzogen haben (beispielsweise Henryk M. Broder, Jan Fleischhauer und Klaus Rainer Röhl). Hinzu kommen die „Unsortierbaren", die sich aufgrund uneinheitlicher Positionen kaum einer bestimmten politischen Heimat zuordnen lassen (etwa Ralph Giordano). Umso verwirrender ist der Buchtitel, der einige Überschneidungen zu „Mahler, Maschke \& Co.“ erwarten lässt. Doch schon das Inhaltsverzeichnis klärt darüber auf, dass sich keiner der Protagonisten von „Die Achse der Abtrünnigen“ am rechten Rand befindet.

Carinis Auswahl zeigt eine weitere Besonderheit des Buchs: Die Heterogenität erstreckt sich nicht allein auf die politischen Ziele, denen die Renegaten nach ihrem Bruch mit links zugeströmt sind, sondern gleichfalls auf die abgedeckte Zeitspanne (das gesamte 20. und den Anfang des 21. Jahrhunderts) und auf die unterschiedlichen politischen Kontexte der Renegaten (Kaiserreich, Weimarer Republik, Nationalsozialismus, Sowjetunion, Bundesrepublik, DDR). Darum taugen die Kurzbiografien, die im ersten Teil des Buches weitgehend kommentarlos aneinandergereiht werden, nicht für einen Vergleich. Überhaupt sucht der Leser vergebens ein Kapitel, das die Essenz der vielen Lebensläufe aufzufangen versucht. Carinis knappe Leitfrage, die er in der Einleitung formuliert, verhallt ungehört. Dabei gibt sich keinem unmöglichen Unterfangen hin, wer eine Klammer um derart verschiedene Biografien, wie etwa die von Jan Fleischhauer und Karl I. Albrecht, ziehen will. Er muss 
allerdings auf einer abstrakteren Ebene arbeiten und systematischer vorgehen als Carini. Dadurch kommt hier der Verdacht der Beliebigkeit auf. Zudem: Wer „Unter Linken“ ähnlich gewichtet wie „Die Revolution entlässt ihre Kinder“, handelt sich den Vorwurf ein, es fehle an analytischer Distanz gegenüber den Renegaten der Gegenwart.

Die Frage, wie es zu den politischen Lagerwechseln kam, motiviert vermutlich am ehesten zum Griff nach den beiden Büchern. Umso enttäuschender fällt allerdings deren Antwort aus: Seitenbecher will bei den fünf Renegaten - abgesehen vom Motiv der „Enttäuschung über einstige Ideale“ der „68er“- je individuelle Entscheidungen identifiziert haben (S. 388) - Maschke sei von Kuba desillusioniert worden, Fichter habe der Linksterrorismus abgestoßen, Oberlercher und Rabehl sähen sich in ihrer Karriere verhindert und Mabler habe auf eigene Faust mit Marx und Lenin gebrochen, um sich anschließend der HegelLektüre zu widmen. Warum dann aber gleich das andere Extrem? Warum haben sie, als sie sich getäuscht sahen, ihre neue politische Heimat am rechten Rand gefunden und nicht im demokratischen Spektrum? Die Antwort auf diese Fragen bleibt Seitenbecher ebenso schuldig wie Erklärungen der Gründe für Fichters politische Mäßigung - wenn er ihn schon in seine Untersuchung einbezieht. Dabei lüde gerade der Sonderfall zum genaueren Hinsehen ein.

Marco Carini wiederum versucht sich erst gar nicht an einer Antwort auf die Frage nach den generellen Ursachen der politischen Lagerverschiebungen, wenngleich sie ja den Anlass dafür bieten, die unterschiedlichen Biografien in einem Buch zu vereinigen. Er verliert sich stattdessen in den Details der Lebensverläufe (erster Teil) und dann in denjenigen Themen, welche die Renegaten nach ihrem „Wandel“ umgetrieben haben (zweiter Teil). Insofern bleibt er deutlich weiter hinter seinem Anspruch zurück als Seitenbecher.

Dessen Antworten hinterlassen beim Leser, der sich Einsichten in generelle sozialpsychologische Abläufe verspricht, allerdings ein unbefriedigendes Gefühl. Ihm gelingt es nicht, seine - instruktiven - Befunde zu abstrahieren. Stattdessen bleibt er nah am Gegenstand und verfolgt im Ganzen eher einen idiografischen Ansatz. Die durch die Aufnahme von Fichter entstandene Heterogenität der Fälle versperrt den Blick auf Parallelen und generelle Muster zusätzlich. Für eine Theorie, die (radikale) politische Lagerwechsel bei den fünf Beispielen oder sogar ganz allgemein zu erklären in der Lage ist, wäre eine theoriegeleitete, systematische Vorgehensweise geboten gewesen. Sie hätte den biografischen Ansatz Seitenbechers, der den Boden der historischen Disziplin zu keiner Zeit verlässt, ergänzen und eine klaffende Lücke der Extremismusforschung schließen können.

Was für Seitenbecher spricht: Er enthüllt den Widerspruch zwischen den historischen Fakten einerseits und der retrospektiven Vereinnahmung von 1968 andererseits. Und er arbeitet die ideologischen und persönlichen Konstanten heraus, die bei einem flüchtigen Blick nicht ins Auge stechen: Schließlich dominiert der Eindruck eines politischen Lagerwechsels. Außerdem überträgt er zu keinem Zeitpunkt seine Befunde vorschnell auf politische Konvertiten anderer Zeit - etwa Benito Mussolini oder Robert Michels - oder wartet mit Küchenpsychologie auf. Seine flüssig geschriebene Studie dürfte als Ausgangspunkt für theoriebildende Arbeiten dienen, die über die fünf Alt-68er hinausweisen. Sie füllt eine der vielen Lücken, die sich demjenigen auftun, der das „Zeitalter der Extreme“ unterhalb der in den Sozialwissenschaften verbreiteten Makroperspektive verstehen will.

„Die Achse der Abtrünnigen“ hingegen dürfte sich vor allem für jene eignen, die mehr erfahren wollen über deutsche Publizisten, die im Laufe ihres Lebens nach rechts gerückt sind. Das überspannende Thema ist - anders als bei Seitenbechers Studie und entgegen dem 
Titel - eben nicht der Bruch mit einer linksextremen Ideologie, sondern die relative politische Positionsverschiebung nach rechts, die mal als Bruch mit einer kommunistischen Partei unter Beibehaltung kommunistischer Ideale, mal als völlige Abkehr von kommunistischen, sozialistischen und sozialdemokratischen Ideen, mal als Entfernung vom linken Lebensmilieu daherkommt. Diese Klammer nicht gefunden zu haben, ist die Hauptschwäche des Bandes, der im Großen und Ganzen auf einer oberflächlichen, deskriptiven Ebene verweilt, sich aber aufgrund des flotten Schreibstils liest wie ein guter Roman.

Tom Mannewitz

Der „sächsische Weg“: Eckstein der vergleichenden Bundesländerforschung

Jesse, Eckhard, Thomas Schubert und Tom Thieme: Politik in Sachsen, Springer VS, Wiesbaden 2014, 349 Seiten, $€ 29,99$.

Die jüngste Untersuchung zum Freistaat Sachsen bereichert den Forschungsstand zu länderspezifischen Regionalstudien um einen weiteren Beitrag. Eckhard Jesse, Professor für Politische Systeme und Politische Institutionen an der Universität Chemnitz, und seine Mitarbeiter Thomas Schubert und Tom Thieme erläutern ihr Grundmotiv wie folgt: „Ziel unseres Buches ist es, das politische System des Freistaates Sachsens in seiner Struktur und Entwicklung zu beschreiben, zu analysieren, vergleichend einzuordnen sowie zu bewerten " (S. 13). Über die Erschließung sächsischer Spezifika soll dadurch die Frage beantwortet werden, ob „mit Blick auf das politische System Sachsens und dessen Kernbestandteile ein ,sächsischer Weg““ (S. 13) existiert. Dieser 2006 von Ulrich Brümmer eingeführte Begriff legt in seiner ursprünglichen Verwendung einen Schwerpunkt auf strukturelle Merkmale der sächsischen Politik seit 1989/90. Diese „Verengung“ (S. 14) möchten die Autoren in ihrer Darstellung überwinden und den Begriff um die prozessualen Komponenten des Politischen erweitern.

In vierzehn Kapiteln soll dadurch ein „facettenreiches Bild, das weder idealisiert noch nivelliert“ (S. 14) gezeichnet werden. Der „sächsische Weg“ setzt sich aus unterschiedlichen Bestandteilen zusammen, die über den Ost-West- und den ostdeutschen Ländervergleich zu Tage treten sollen. Für die Autoren ist diese Untersuchung ergebnisoffen (S. 15), doch jede „im Ländervergleich markante, temporär bestandsfeste und kontextuell sächsische Eigenheit“ (S. 15) bietet den Verfassern Ansatzpunkte. „Gewiss, jedes Land hat Spezifika, aber Sachsen weist ,besondere` auf. Insofern ist die Frage nach dem ,sächsischen Weg' nicht künstlich hergeholt, eine gründliche Antwort vielmehr sinn- und reizvoll“ (S. 30).

Die Autoren können ihrem eigenen Anspruch standhalten, und es gelingt eine detaillierte Beschreibung struktureller und prozessualer Komponenten des politischen Systems Sachsens. Der Begriff des „sächsischen Weges“ bleibt dabei stets präsent und zieht sich nach der Einleitung durch alle vierzehn Kapitel (Wiedergründung 1989/90, Verfassung und Justiz, Landtag, Landesregierung, die Rolle der Ministerpräsidenten, Wahlen und Wahlverhalten, Parteien und Parteiensystem, Verwaltung, die kommunale Ebene, Einfluss auf Bundes- und europäischer Ebene, Medien, Demografie, regionale politische Kultur sowie „Politischer Extremismus und Demokratieschutz“). Die Kapitel sind annähernd gleich untergliedert, 